\title{
Red density perturbations and inflationary gravitational waves
}

\author{
Luca Pagano $^{1}$, Asantha Cooray ${ }^{2}$, Alessandro Melchiorri ${ }^{1,3}$ \\ and Marc Kamionkowski ${ }^{4}$ \\ ${ }^{1}$ Physics Department and Sezione INFN, University of Rome 'La Sapienza', \\ Piazzale Aldo Moro 2, I-00185 Rome, Italy \\ 2 Department of Physics and Astronomy, University of California, Irvine, \\ CA 92697, USA \\ ${ }^{3}$ CERN, Theory Division, Geneva 23, CH-1211, Switzerland \\ ${ }^{4}$ California Institute of Technology, Mail Code 130-33, Pasadena, \\ CA 91125, USA \\ E-mail: luca.pagano@roma1.infn.it, acooray@uci.edu, \\ alessandro.melchiorri@roma1.infn.it and kamion@tapir.caltech.edu
}

Received 13 January 2008

Accepted 14 March 2008

Published 7 April 2008

Online at stacks.iop.org/JCAP $/ 2008 / \mathrm{i}=04 / \mathrm{a}=009$

doi:10.1088/1475-7516/2008/04/009

\begin{abstract}
We study the implications of recent indications from the Wilkinson Microwave Anisotropy Probe (WMAP) and other cosmological data for a red spectrum of primordial density perturbations for the detection of inflationary gravitational waves (IGWs) with forthcoming cosmic microwave background experiments. We consider a variety of single-field power-law, chaotic, spontaneous symmetry-breaking and Coleman-Weinberg inflationary potentials which are expected to provide a sizable tensor component and quantify the expected tensorto-scalar ratio given existing constraints from WMAP on the tensor-to-scalar ratio and the power spectrum tilt. We discuss the ability of the near-future Planck satellite to detect the IGW background in the framework of those models. We find that the proposed satellite missions of the Cosmic Vision and Inflation Probe programs will be able to detect IGWs from all the models we have surveyed at better than $5 \sigma$ confidence level. We also provide an example of what is required if the IGW background is to remain undetected even by these latter experiments.
\end{abstract}

Keywords: CMBR experiments, CMBR detectors, CMBR polarization parameters, inflation 


\section{Contents}

1. Introduction 2

2. Inflationary potentials and tensor-to-scalar ratio 4

3. Results and discussion 5

$\begin{array}{ll}\text { Acknowledgments } & 9\end{array}$

$\begin{array}{ll}\text { References } & 9\end{array}$

\section{Introduction}

Inflation's [1] predictions of a flat Universe and a nearly Gaussian and nearly scaleinvariant spectrum of primordial density perturbations [2] have been confirmed by a suite of recent cosmic microwave background (CMB) experiments $[3,4,6]$. A third prediction of inflation is a stochastic background of inflationary gravitational waves (IGWs) [7]. The theory predicts that the IGW amplitude is proportional to the cosmological energy density during inflation. Since this energy density varies from model to model, the IGW amplitude cannot be predicted in a model-independent way. If, however, IGWs can be detected, they will provide an important probe of the physics of inflation.

The CMB polarization is perhaps the most promising tool for detecting the IGW background. The statistical properties of CMB linear polarization are fully characterized by two sets of spin-2 multipole moments with opposite parities [8]. The magnetic-type modes (B or curl modes) are produced by IGWs (or 'tensor' metric perturbations) and not density perturbations (scalar metric perturbations), and they do not correlate with the temperature nor the electric-type-parity modes ( $\mathrm{E}$ or grad modes). A detection of B-mode polarization would thus provide good evidence for IGWs [9].

The scalar and tensor perturbations generated by inflation have power spectra that are generally well approximated by power laws: $P_{\mathrm{s}}(k) \propto k^{n_{\mathrm{s}}}$ and $P_{\mathrm{t}}(k) \propto k^{n_{\mathrm{t}}}$, respectively, as a function of the spatial wavenumber $k$. The amplitudes and spectral indices can be written in terms of the inflaton potential $V(\phi)$ and its first and second derivatives $V^{\prime}(\phi)$ and $V^{\prime \prime}(\phi)$. More precisely, the spectral indices $n_{\mathrm{s}}$ and $n_{\mathrm{t}}$ can be written in terms of the slow-roll parameters $\epsilon$ and $\eta[10]$ :

$$
n_{\mathrm{s}} \simeq 1-6 \epsilon+2 \eta, \quad n_{\mathrm{t}} \simeq-2 \epsilon,
$$

with

$$
\epsilon \equiv \frac{m_{\mathrm{Pl}}^{2}}{16 \pi}\left(\frac{V^{\prime}(\phi)}{V(\phi)}\right)^{2}, \quad \eta \equiv \frac{m_{\mathrm{Pl}}^{2}}{8 \pi} \frac{V^{\prime \prime}(\phi)}{V(\phi)}
$$

where $m_{\mathrm{Pl}}$ is the reduced Planck mass. The observed density-perturbation amplitude already fixes $V / \epsilon \simeq 6.6 \times 10^{16} \mathrm{GeV}$. The tensor-to-scalar ratio $r$ is $r=16 \epsilon$. Since $n_{\mathrm{t}}$ and $r$ depend only on $\epsilon$, they satisfy a consistency relation $n_{\mathrm{t}} \simeq-2 \epsilon=-r / 8$ that can be tested with measurements, if the IGW background can be detected. 
An important new result from recent $\mathrm{CMB}$ experiments is that a value $n_{\mathrm{s}}=1$ now seems unlikely; the likelihood for its value peaks closer to $n_{\mathrm{s}}=0.95[4,11]$. Although it is still debated whether the data are inconsistent with $n_{\mathrm{s}}=1$ at the $2 \sigma$ level or at the $3 \sigma$ level [12], it is clear that a value $n_{\mathrm{s}}=1$ is becoming increasingly difficult to reconcile with the data. Although a measurement of $n_{\mathrm{s}}$ does not uniquely determine $\epsilon$, and that $n_{\mathrm{s}}<1$ does not provide per se any evidence for a sizable tensor contribution, in several inflationary models $\epsilon$ and $\eta$ are roughly expected of the same magnitude, or perhaps $\epsilon$ is even larger than $\eta$ (if, for example, the inflaton potential can be approximated as linear). If $\epsilon \gtrsim \eta$ then a value $n_{\mathrm{s}} \simeq$ 0.95-0.99 may suggest $\epsilon=\mathcal{O}(0.01)$, which thus implies a $r \sim 0.1$, perhaps within the range of detectability with next-generation experiments, as argued similarly in [13].

It is therefore timely to discuss the ability of near-(and far-) future satellite missions to detect the IGW signal if inflation is described by one of the above models.

The purpose of this paper is indeed to quantify the feasibility for future detection by considering a variety of functional forms for $V(\phi)$, consistent with current data and able to provide a sizable tensor contribution. In particular, we will address the level of significance of the detection and the percentage of detectable models that belong to a particular potential by marginalizing over different target models. Moreover, we will discuss the improvement that future satellite missions are expected to provide with respect to the Planck satellite experiment, expected to be launched this autumn.

As we will see, the amplitude of the IGW background can be large enough to be detectable by next-generation experiments in a broad family of inflation models consistent with current data. While one can conceive a large number of potential forms for $V(\phi)$, we select classes of models here that are frequently discussed in the literature involving singlefield power-law, chaotic, spontaneous symmetry-breaking potentials and the ColemanWeinberg (CW) potential. The first three models generally have the property that a redder tilt results in a larger $r$, while this is behavior not present with the $\mathrm{CW}$ potential.

This kind of approach could be considered as 'optimistic' in the sense that inflationary models with negligible IGW can be conceived for any value of $n_{\mathrm{s}}$. In this case the only 'measurement' will be an upper limit on $r$ which will provide much less information on the scale of inflation. Analyses on future upper limits on $r$ have already been presented in several papers and we will not repeat this discussion here.

Our analysis follows recent work on this subject in [13] and [14]. Our work, however, differs from these analyses by considering a broad range of models and by using a different technique to forecast the expected amplitude of IGWs in Planck, BPol and EPIC. The experimental configurations we have considered for the last two proposed missions are more consistent with concept studies that are now ongoing to develop them in the USA and Europe.

While our selection of inflationary potentials is limited to four forms, these forms are broad enough that we can make a general statement about the detectability of IGWs with CMB polarization observations. The same potentials are routinely discussed in the literature when describing inflation, and the $\mathrm{CW}$ potential is generally given as an example of an inflationary model that leads to a lower amplitude of the tensor-to-scalar ratio. We find that the power-law, chaotic and SSB models are very likely to be detectable with nextgeneration experiments, unless the power-law model has a very small power-law index. There are indeed CW models that will remain undetectable, even by next-generation 
experiments. However, if we choose a uniform prior in the spectral-index-IGW-amplitude parameter space for these models and we assume that $n \geq 0.94$, then we still find that they are likely to be detected.

\section{Inflationary potentials and tensor-to-scalar ratio}

To establish the expected amplitude of IGWs, we consider four classes of inflationary models [15]-[19]:

(1) Power-law inflation is characterized by an inflaton potential $V(\phi) \propto \mathrm{e}^{\phi / \mu}$, where $\mu$ is a mass scale. In this potential, there is a relation, $r=8\left(1-n_{\mathrm{s}}\right)$, between the tensor-to-scalar ratio and the scalar spectral index.

(2) Chaotic inflation features an inflaton potential $V(\phi) \propto(\phi / \mu)^{p}$. In theoretically attractive models, $p$ is a small integer. Experimentally, $p \lesssim 10$ if $n_{\mathrm{s}} \gtrsim 0.9$ [17], but $p$ can empirically be arbitrarily small. We consider values of $p$ between $p=0.1$ and 8 in our numerical work. In these models, $r=8[p /(p+2)]\left(1-n_{\mathrm{s}}\right)$.

(3) Spontaneous symmetry-breaking $(S S B)$ inflation features an inflaton potential $V(\phi) \propto$ $\left[1-(\phi / \nu)^{2}\right]^{2}$. The precise model is specified by two parameters: $\nu$ and $N_{\mathrm{e}}$, the number of e-foldings of inflation between CMB scales and the end of inflation. A conservative range for $N_{\mathrm{e}}$ is $47 \lesssim N_{\mathrm{e}} \lesssim 62$, corresponding to an inflationary energy scale in the range of an $\mathrm{MeV}$ to $10^{16} \mathrm{GeV}$, the current upper bound. The $n_{\mathrm{s}}-r$ relation for $\mathrm{SSB}$ models cannot be written in a simple way. To obtain it, we use the algorithm given in equations (38)-(41) of [17].

(4) Coleman-Weinberg (CW) inflationary model $[18,19]$, based on a quartic potential of the form $V(\phi) \propto \phi^{4} \ln \left(\phi^{2} / M_{*}^{2}\right)$, where $M_{*}=10^{18} \mathrm{GeV}$ is an arbitrary renormalization mass (see [20]). This potential has been shown to be in agreement with WMAP3 [19].

We show in figure 1 the corresponding regions in the $n_{\mathrm{s}}-r$ parameter space for the above four models and with different values of $p$ in the case of chaotic models. In addition to these analytical descriptions of $V(\phi)$, one could also consider hybrid inflation models, which generally feature multiple fields. Their phenomenology can usually be modeled, though, as a single field with the addition of a non-zero cosmological constant at the inflaton potential minimum. What distinguishes these models phenomenologically is that they usually produce $n_{\mathrm{s}}>1$. Since the working assumption of this paper is that $n_{\mathrm{s}}<1$, as suggested by WMAP data, we do not consider these models further.

In their analysis [4] of their three-year data, the Wilkinson microwave anisotropy probe (WMAP) collaboration surveyed the cosmological parameter space assuming $n_{\mathrm{s}}, r$ and $n_{\mathrm{t}}$ to be independent parameters. The resulting constraints to the $n_{\mathrm{s}}-r$ parameter space are shown in figure 1. However, if inflation is responsible for primordial perturbations, and if the inflaton potential is power-law, chaotic, SSB or CW then there is a fixed relation between $n_{\mathrm{s}}, r$ and $n_{\mathrm{t}}$, i.e. they are not independent parameters.

We therefore re-analyze the CMB data for the power-law, chaotic, SSB and CW models, imposing the consistency relations between $n_{\mathrm{s}}, r$ and $n_{\mathrm{t}}$ obtained from the above potentials. We also include a prior $r \geq 10^{-5}$ which is below the minimum amount of tensor modes detectable by future experiments. We use the Markov chain Monte Carlo (MCMC) package cosmomcmc [21] to run a set of chains, imposing these consistency relations. We 


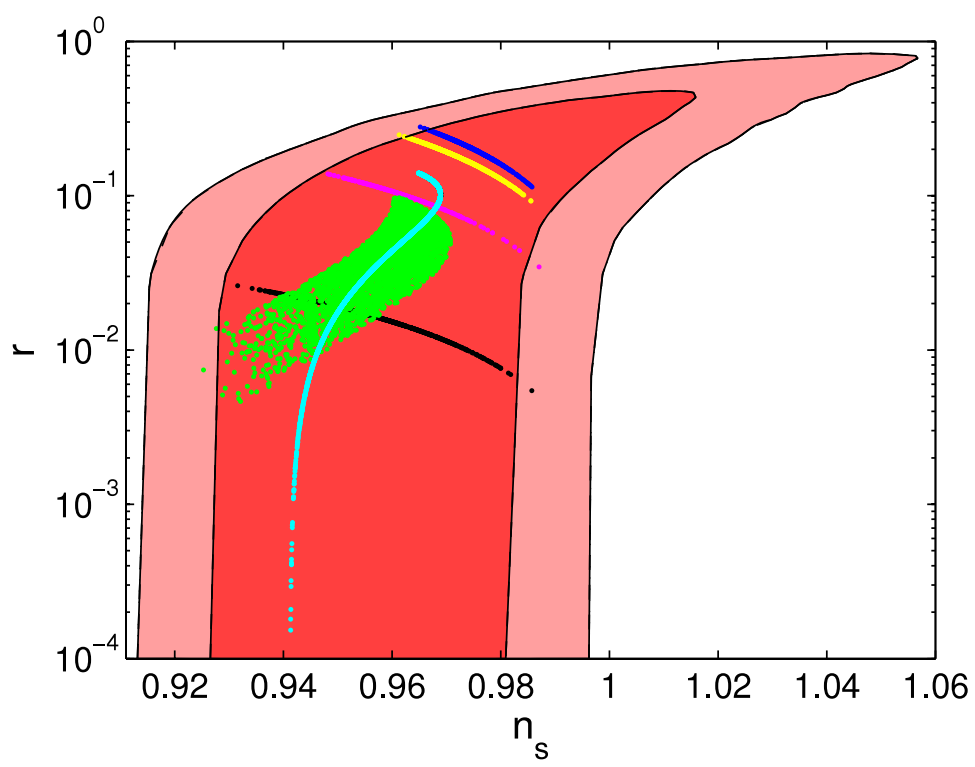

Figure 1. The WMAP (red shaded areas) constraints to the $n_{\mathrm{s}}-r$ parameter space. The outer (inner) contours are the $2 \sigma(1 \sigma)$ contours. We also plot the models within $\Delta \chi^{2}=1(1 \sigma)$ from the best fit for power-law and chaotic models, and at $\Delta \chi^{2}=2.3$ (also $1 \sigma$ ) for the SSB models. The curves, from top to bottom, are for power-law and for chaotic $p=8,1,0.1$ and Coleman-Weinberg. The (green) points are for the SSB models.

Table 1. The $68 \%$ C.L. limits on the spectral index $n_{\mathrm{s}}$ and the scalar-tensor ratio $r$ from WMAP, assuming the $n_{\mathrm{s}}-r$ relation for each class of inflationary models.

\begin{tabular}{lll}
\hline Model & $n_{\mathrm{s}}$ & $r$ \\
\hline Power-law & $0.980_{-0.005}^{+0.005}$ & $0.16_{-0.04}^{+0.04}$ \\
Chaotic $p=1$ & $0.970_{-0.008}^{+0.008}$ & $0.080_{-0.041}^{+0.020}$ \\
Chaotic $p=8$ & $0.978_{-0.011}^{+0.011}$ & $0.14_{-0.07}^{+0.07}$ \\
Chaotic $p=0.1$ & $0.964_{-0.018}^{+0.019}$ & $0.014_{-0.007}^{+0.004}$ \\
SSB $\left(N_{\mathrm{e}}=47-62\right)$ & $0.957_{-0.020}^{+0.006}$ & $0.042_{-0.014}^{+0.010}$ \\
$\mathrm{CW}$ & $0.953_{-0.012}^{+0.013}$ & $0.052_{-0.026}^{+0.013}$ \\
\hline
\end{tabular}

use only the latest WMAP results [4]. The likelihood is determined using the October 2006 version of the WMAP likelihood code available at [5]. The likelihoods are obtained after marginalizing (with flat priors) over the baryon and cold-dark-matter densities, the ratio of the sound horizon to the angular-diameter distance at decoupling and the optical depth to reionization.

\section{Results and discussion}

In table 1 , we list the current constraints to $n_{\mathrm{s}}$ and $r$ from WMAP obtained using each class of inflationary models as a prior. As expected, the tabulated values and the plots indicate that tensor modes are required for exponential, power-law and small-field scenarios. 


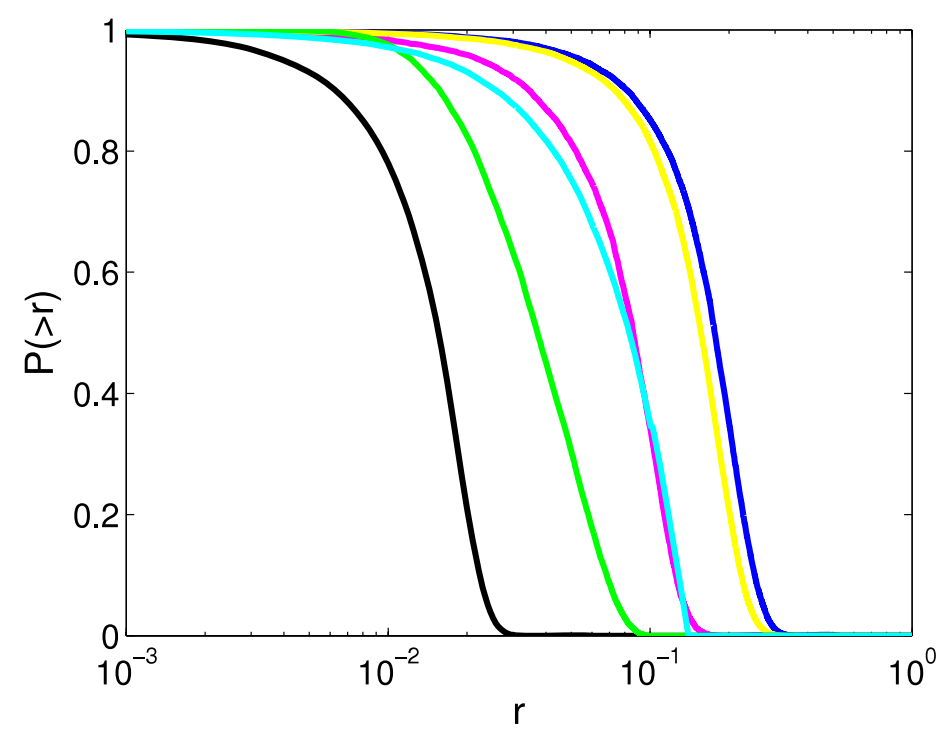

Figure 2. Percentage of inflationary models with a tensor-to-scalar ratio above a threshold value $r$, for the various inflaton potential classes considered in figure 1 . From right to left, the models are power-law, chaotic $p=8$, chaotic $p=1$, Coleman-Weinberg, SSB and chaotic $p=0.1$.

Our analysis provides a likelihood, from CMB data, for each point along the curves associated with each class of inflationary models. We can thus plot in figure 2 the percentage of models with a tensor amplitude above a threshold value $r$. As shown, almost all of the exponential and power-law models have a tensor amplitude $r>2 \times 10^{-2}$, while small-field models predict $r>2 \times 10^{-3}$. The CW model predicts a sizable tensor contribution of $r \sim 0.1$ for $n_{\mathrm{s}} \sim 0.965$, but it vanishes for $n_{\mathrm{s}}<0.94$ and $n_{\mathrm{s}}>0.98$ (see also figure 3 in [19]).

If experiments were to probe values of $r$ well below $2 \times 10^{-3}$ without finding any evidence for gravitational waves, then it would rule out a large class of single-field inflationary models.

Previous work [22] can already be used to estimate the fraction of models in each class of models that can be detected by a given experiment. Here we go further by considering values of $n_{\mathrm{t}} \neq 0$ that are given by the inflationary consistency relation. We also evaluate the likelihood, given current WMAP constraints to each class of inflationary models (e.g. power-law, chaotic, SSB), that IGWs will be detected by a particular future experiment.

We begin by evaluating, for a given future experiment and for a given set of inflationary parameters, the corresponding Fisher matrix [23]:

$$
F_{i j}=\sum_{l=2}^{l_{\max }} \sum_{\alpha, \beta} \frac{\partial C_{l}^{\alpha}}{\partial \theta_{i}}\left(\operatorname{Cov}_{l}\right)_{\alpha \beta}^{-1} \frac{\partial C_{l}^{\beta}}{\partial \theta_{j}},
$$

where the $C_{l}^{\alpha \beta}$ are the power spectra for the temperature (TT), temperature polarization (TE), E-mode polarization (EE) and B-mode polarization ( $\alpha$ and $\beta$ run over TT, EE, TE and $\mathrm{BB}), \theta_{i}$ is the set of parameters used in the Markov chain and $\operatorname{Cov}_{l}$ is the spectra covariance matrix' [8]. The covariance-matrix entries depend on the instrumental noise 
Table 2. Experimental specifications for the satellite missions considered in this work. Channel frequency is given in $\mathrm{GHz}, \mathrm{FWHM}$ in arcminutes and noise in $10^{-6}$.

\begin{tabular}{lllll}
\hline Experiment & Chan. & FWHM & $\Delta T / T$ & $\Delta P / T$ \\
\hline WMAP & 40 & $28^{\prime}$ & 8.2 & 11.6 \\
$f_{\text {sky }}=0.65$ & 60 & $21^{\prime}$ & 11.0 & 15.6 \\
& 90 & $13^{\prime}$ & 18.3 & 25.9 \\
Planck & 44 & $23^{\prime}$ & 2.7 & 3.9 \\
$f_{\text {sky }}=0.65$ & 70 & $14^{\prime}$ & 4.7 & 4.7 \\
& 100 & $9.5^{\prime}$ & 2.5 & 4.0 \\
& 143 & $7.1^{\prime}$ & 2.2 & 4.2 \\
& 217 & $5.0^{\prime}$ & 4.8 & 9.8 \\
BPol & 353 & $5.0^{\prime}$ & 14.7 & 29.8 \\
$f_{\text {sky }}=0.65$ & 70 & $30^{\prime}$ & 0.12 & 0.24 \\
& 100 & $30^{\prime}$ & 0.062 & 0.12 \\
& 143 & $30^{\prime}$ & 0.055 & 0.11 \\
EPIC & 217 & $30^{\prime}$ & 0.046 & 0.092 \\
$f_{\text {sky }}=0.65$ & 40 & $30^{\prime}$ & 0.062 & 0.12 \\
& 90 & $116^{\prime}$ & 0.032 & 0.047 \\
& 90 & $52^{\prime}$ & 0.018 & 0.039 \\
& 135 & $34^{\prime}$ & 0.0077 & 0.025 \\
& & & &
\end{tabular}

and the angular resolution of the experiment. The $1 \sigma$ (or $68 \%$ C.L.) error on a particular parameter $\theta_{j}$ is $\sigma\left(\theta_{j}\right)=\left(F^{-1}\right)_{j j}^{1 / 2}[23]$, after marginalizing over other undetermined parameters.

Now consider a particular class (e.g. power-law, chaotic or SSB) of inflationary models. The probability that IGWs will be detected at $N \sigma$, given the current WMAP constraints, is then

$$
P=\sum_{i=1}^{M} \Theta\left[r_{i}-N \sigma\left(r_{i}\right)\right] / M,
$$

where $\Theta$ is the Heaviside step function. The sum is over the $M$ models consistent at $95 \%$ C.L. with WMAP assuming one of the class of inflationary models. For example, if $P=1$ for $N=2$, then the future experiment will detect IGWs with $2 \sigma$ significance from all models compatible with WMAP within that particular class of models.

We consider here four experimental configurations: eight years of WMAP; the Planck satellite [24] (to be launched in 2008); and two proposed next-generation satellites, BPol [25] and EPIC [26]. Since the amplitude of the galactic foregrounds for polarization is still not determined by observations, we remove channels below $40 \mathrm{GHz}$ and above $250 \mathrm{GHz}$, as these are likely to be contaminated by synchrotron and dust emission, respectively. The parameters assumed for these missions are summarized in table 2.

Our results are reported in table 3 for detections with $2 \sigma$ and $5 \sigma$ significance. Our results suggest that eight years of WMAP data are unlikely to detect IGWs. The Planck satellite, on the other hand, stands a good chance to measure the IGW background in power-law models, even at the $5 \sigma$ confidence level. This forecast, however, may be 
Table 3. Percentage of models in agreement with the WMAP observations and with an IGW background detectable at $2 \sigma$ and $5 \sigma$ confidence levels by the experimental configurations listed in table 2.

\begin{tabular}{lllll}
\hline Model & WMAP8yr & Planck 6ch & BPol & Epic \\
\hline Power-law & $0.45 / 0$ & $1 / 0.98$ & $1 / 1$ & $1 / 1$ \\
Chaotic $p=1$ & $0 / 0$ & $0.99 / 0.67$ & $1 / 1$ & $1 / 1$ \\
Chaotic $p=8$ & $0.30 / 0$ & $1 / 0.97$ & $1 / 1$ & $1 / 1$ \\
Chaotic $p=0.1$ & $0 / 0$ & $0.60 / 0$ & $1 / 1$ & $1 / 1$ \\
SSB $\left(N_{\mathrm{e}}=47-62\right)$ & $0 / 0$ & $0.78 / 0.09$ & $1 / 1$ & $1 / 1$ \\
CW & $0 / 0$ & $0.83 / 0.42$ & $0.92 / 0.92$ & $0.92 / 0.92$ \\
\hline
\end{tabular}

Table 4. Planck only. Percentage of models in agreement with the WMAP observations and with an IGW background detectable at $2 \sigma / 5 \sigma$ by the Planck experiment as a function of the channels available for cosmology. The $T+C$ does not include the polarization channels but just the temperature and the crosstemperature-polarization spectrum.

\begin{tabular}{llll}
\hline Model & 4ch & 100 GHzch & Allch $T+C$ \\
\hline Power-Law & $1 / 0.97$ & $0.99 / 0.30$ & $0.81 / 0.02$ \\
Chaotic $p=1$ & $0.99 / 0.61$ & $0.92 / 0$ & $0.25 / 0$ \\
Chaotic $p=8$ & $1 / 0.96$ & $0.99 / 0.16$ & $0.81 / 0$ \\
Chaotic $p=0.1$ & $0.50 / 0$ & $0 / 0$ & $0 / 0$ \\
SSB $\left(N_{\mathrm{e}}=62-47\right)$ & $0.77 / 0.07$ & $0.36 / 0$ & $0 / 0$ \\
CW & $0.81 / 0.35$ & $0.57 / 0$ & $0.21 / 0$ \\
\hline
\end{tabular}

optimistic, since it assumes perfect foreground removal and the full use of six channels for cosmology. To understand these effects better, we show forecasts in table 4 for Planck, assuming first a smaller number of channels and then a more pessimistic configuration where no EE or BB power spectra are used. While we found only a small degradation in decreasing the number of channels from 6 to 4, using one channel only, or just using TT and TE spectra alone, will not be able to produce more than an indication for tensor modes. With either an Inflation Probe or a Cosmic Vision mission for high-precision CMB polarization measurements, we find that the full set of single-field inflationary models we have considered can be explored.

If no tensor modes are detected after one of the planned post-Planck CMB polarization missions, then it is very likely that inflation should be described by a different class of models than we have considered, or perhaps that the measure we chose for the $\mathrm{CW}$ models was inappropriate. In this case, we note that generic potentials of the form $V(\phi) \sim\left[1-(\phi / \nu)^{p}\right]$ with $p=4,6,8, \ldots$ lead to $|\eta| \gg \epsilon$ and to arbitrary small values of $r$. Hybrid inflation models with a logarithmic potential such as $V(\phi) \sim[1+\alpha \ln (\phi)]$ motivated, for example, by SUSY models with broken supersymmetry may also have $n_{\mathrm{s}}<1$ and a completely arbitrary small value for $r$. Alternatives could then be even more complicated models, such as chaotic inflation with an unusually small $p(p \lesssim 0.01)$, models with multiple fields, string models or models with extra dimensions. Finally, many other and well physically motivated mechanisms such as topological defects may give rise to an extra IGW background that would completely change our results. 
Inflationary gravitational waves

\section{Acknowledgments}

We thank W Kinney for useful discussions and comments on an earlier draft. AC acknowledges support from NSF CAREER AST-0645427. MK acknowledges support from DoE DE-FG03-92-ER40701, NASA NNG05GF69G, and the Gordon and Betty Moore Foundation.

\section{References}

[1] Guth A H, 1981 Phys. Rev. D 23347 [SPIRES] Albrecht A and Steinhardt P J, 1982 Phys. Rev. Lett. 481220 [SPIRES] Linde A D, 1982 Phys. Lett. B 108389 [SPIRES]

[2] Guth A H and Pi S Y, 1982 Phys. Rev. Lett. 491110 [SPIRES] Hawking S W, 1982 Phys. Lett. B 115295 [SPIRES] Linde A D, 1982 Phys. Lett. B 116335 [SPIRES] Starobinsky A A, 1982 Phys. Lett. B 117175 [SPIRES] Bardeen J M, Steinhardt P J and Turner M S, 1983 Phys. Rev. D 28679 [SPIRES] Mukhanov V F and Chibisov G V, 1981 Pis. Zh. Eksp. Teor. Fiz. 33549 Mukhanov V F and Chibisov G V, 1981 JETP Lett. 33532 (translation)

[3] Lange A E et al (Boomerang Collaboration), 2001 Phys. Rev. D 63042001 [SPIRES] [astro-ph/0005004] Miller A D et al, 1999 Astrophys. J. 524 L1 [SPIRES] [astro-ph/9906421] Hanany S et al, 2000 Astrophys. J. 545 L5 [SPIRES] [astro-ph/0005123] Halverson N W et al, 2002 Astrophys. J. 56838 [SPIRES] [astro-ph/0104489] Mason B S et al, 2003 Astrophys. J. 591540 [SPIRES] [astro-ph/0205384] Benoit A et al (the Archeops Collaboration), 2003 Astron. Astrophys. 399 L25 [SPIRES] [astro-ph/0210306]

Goldstein J H et al, 2003 Astrophys. J. 599773 [SPIRES] [astro-ph/0212517]

[4] Spergel D N et al (WMAP Collaboration), 2006 Preprint astro-ph/0603449

[5] http://lambda.gsfc.nasa.gov

[6] Kinney W H, Kolb E W, Melchiorri A and Riotto A, 2006 Phys. Rev. D 74023502 [SPIRES] [astro-ph/0605338]

[7] Abbott L F and Wise M B, 1984 Nucl. Phys. B 244541 [SPIRES] Starobinsky A A, 1979 Pis. Zh. Eksp. Teor. Fiz. 30719 Starobinsky A A, 1979 JETP Lett. 30682 (translation) Rubakov V A, Sazhin M V and Veryaskin A V, 1982 Phys. Lett. B 115189 [SPIRES] Fabbri R and Pollock M D, 1983 Phys. Lett. B 125445 [SPIRES]

[8] Zaldarriaga M and Seljak U, 1997 Phys. Rev. D 551830 [SPIRES] [astro-ph/9609170] Kamionkowski M, Kosowsky A and Stebbins A, 1997 Phys. Rev. D 557368 [SPIRES] [astro-ph/9611125]

[9] Kamionkowski M, Kosowsky A and Stebbins A, 1997 Phys. Rev. Lett. 782058 [SPIRES] [astro-ph/9609132]

Seljak U and Zaldarriaga M, 1997 Phys. Rev. Lett. 782054 [SPIRES] [astro-ph/9609169]

[10] Kamionkowski M and Kosowsky A, 1999 Ann. Rev. Nucl. Part. Sci. 4977 [SPIRES] [astro-ph/9904108]

[11] MacTavish C J et al, 2006 Astrophys. J. 647799 [SPIRES] [astro-ph/0507503]

[12] Huffenberger K M, Eriksen H K and Hansen F K, 2006 Astrophys. J. 651 L81 [SPIRES] [astro-ph/0606538]

[13] Boyle L A, Steinhardt P J and Turok N, 2006 Phys. Rev. Lett. 96111301 [SPIRES] [astro-ph/0507455]

[14] Boyanovsky D, de Vega H J, Ho C M and Sanchez N G, 2007 Phys. Rev. D 75123504 [SPIRES] [astro-ph/0702627]

[15] Dodelson S, Kinney W H and Kolb E W, 1997 Phys. Rev. D 563207 [SPIRES] [astro-ph/9702166]

[16] Kinney W H, 1998 Phys. Rev. D 58123506 [SPIRES] [astro-ph/9806259]

[17] Smith T L, Kamionkowski M and Cooray A, 2006 Phys. Rev. D 73023504 [SPIRES] [astro-ph/0506422]

[18] Kinney W H and Mahanthappa K T, 1996 Phys. Lett. B 38324 [SPIRES] [hep-ph/9511460]

[19] Shafi Q and Senoguz V N, 2006 Phys. Rev. D 73127301 [SPIRES] [astro-ph/0603830]

[20] Shafi Q and Vilenkin A, 1984 Phys. Rev. Lett. 52691 [SPIRES]

[21] Lewis A and Bridle S, 2002 Phys. Rev. D 66103511 [SPIRES] [astro-ph/0205436] (available from http://cosmologist.info)

[22] Kamionkowski M and Kosowsky A, 1998 Phys. Rev. D 57685 [SPIRES] [astro-ph/9705219] Jaffe A H, Kamionkowski M and Wang L M, 2000 Phys. Rev. D 61083501 [SPIRES] [astro-ph/9909281] Lewis A, Challinor A and Turok N, 2002 Phys. Rev. D 65023505 [SPIRES] [astro-ph/0106536] 
Kesden M, Cooray A and Kamionkowski M, 2002 Phys. Rev. Lett. 89011304 [SPIRES] [astro-ph/0202434] Knox L and Song Y S, 2002 Phys. Rev. Lett. 89011303 [SPIRES] [astro-ph/0202286]

Seljak U and Hirata C M, 2004 Phys. Rev. D 69043005 [SPIRES] [astro-ph/0310163]

Verde L, Peiris H and Jimenez R, 2006 J. Cosmol. Astropart. Phys. JCAP01(2006)019 [SPIRES] [astro-ph/0506036]

[23] Jungman G et al, 1996 Phys. Rev. Lett. 761007 [SPIRES] [astro-ph/9507080]

Jungman G et al, 1996 Phys. Rev. D 541332 [SPIRES] [astro-ph/9512139]

[24] Planck Collaboration, 2006 Preprint astro-ph/0604069

[25] http://www.b-pol.org

[26] Bock J et al, 2007 in preparation 